\title{
IOT TECHNOLOGY-BASED SMART DISTRIBUTION FRAMEWORK FOR THE RECOGNITION OF VULNERABILITIES
}

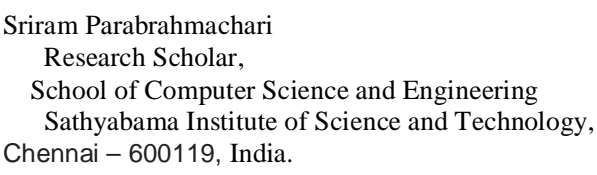

\author{
Dr.N.Srinivasan \\ Sr. Consultant, \\ BAssure Solutions Pvt. Limited \\ Chennai, India.
}

\begin{abstract}
The Internet of Things (IoT) transforms everybody's life through functionality like control and tracking of linked intelligent devices. Smart towns, houses, vehicles, factories, ehealthcare to intelligent control systems, commuting, clothing, agriculture, and many more size IoT applications. The adaptation of these instruments is increasing rapidly, generating considerable data for visualization and interpretation. These systems are also vulnerable to various security risks and issues alongside easy human life, which not only concern consumers for their adoption in sensitive environments such as e-health, smart homes and so on, but also represent a risk for IoT nutrition in the next few days. The Internet of Things (IoT) transforms everybody's life through functions like the tracking and surveillance of the associated intelligent objects. Smart cities, houses, vehicles, factories, e-healthcare, commuting, wearable tech, agriculture and more are all IoT technologies. The adaptation of such instruments is exponentially increasing, which produces considerable data for processing and analysis. These systems are thus vulnerable to various threats and safety challenges alongside facilitating human life, not only causing concern to the consumers for their use in centralized locations including such e-health and intelligent home etc, but also posing a risk to IoT nutrition in the coming days. Moreover, a detailed competitive study between the suggested technique and other related schemes reveals that a better deal is reached with the suggested Method in comparison with other schemes between the safety and accessibility features, connectivity and computing costs.
\end{abstract}

\section{Introduction}

Due to various physical objects that are linked at an unrivalled scale, the IoT is conceivable. This network connection enables these products to interact as well as to share data, including sensors, smart meters, smartphones, smart vehicles and RFID tags, electronic devices (Personal digital assistants (PDA)) and other electronic-integrated items, software and actuators. This is often called an interconnection between physical and virtual objects. Any physical items may include smartphones, sensors, camera, drones, vehicles, etc., whereas virtual items may be called agenda, electronic tickets, books, wallets and so forth [1].

We hope that most of these IoT systems can be intelligent in future, so that devices can take action automatically without any significantly greater human interference. As the Internet communication infrastructure is developed to encircle smart devices, suitable methodologies in areas such as the health care, remote monitoring and monitoring of elderly people, the green economy and vehicle internet (IoV) are very essential for secure communication with these smart devices, and in the future IoT applications. By end 2020, 25 billion appliances will be internet enabled and will be able to study the data used to make 
smart decisions autonomously, according to Gartner's worldwide renowned research and advice company.

However, the aim of IoT remains to allow machines, instruments and nodes to collect, process and make decisions without or at least without human input. IoT building blocks are not formally specified, but can be defined in sensors, computing, connectivity and action plants that generate data for the purpose of gaining information and making smart choices. The data generated by these sensors have brought together diverse areas such as computer science, software engineering, sensing, artificial intelligence, networking and communication. Since IoT progresses rapidly, smarter technologies are imagined goods. Because of the diversity of the IoT environment and the rapid success of huge science, the absence of a formal definition and standardization of IoT has also opened the door. More than $85 \%$ of the world's organisations, according to which about $90 \%$ of the companies are uncertain of IoT's protection, will leverage IoT devices in various ways [28].

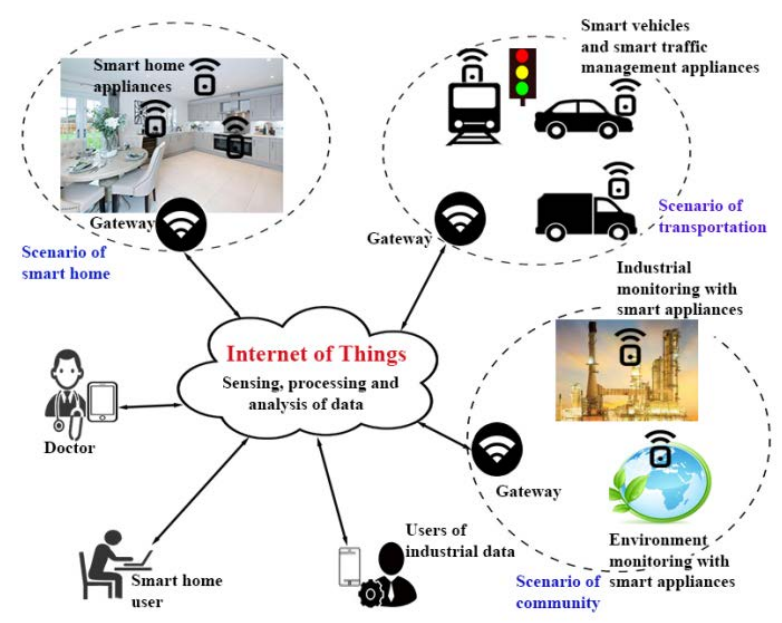

Figure 1. Overview of the IoT environment

Likewise, J. Steinberg et al. state that a large number of intelligent home devices will spy on people at home. A survey conducted by HP shows that $70 \%$ of IoT computers, if attached to the internet, are vulnerable to multiple attacks. In addition, high safety dangers are posed by the recently changed IoT industries such as electricity, shipping, chemical and wastewater systems. Attacks on industrial infrastructure are a fact and no longer a threat after two Russian security researchers identified more than 60,000 bugs that are able to take full control of compromised systems. In addition, by the end of 2020, 25\% of attacks by undertakings will be attributed to infected IoT computers.

The IoT technology has been developed and is widely used in the areas of environmental surveillance, medical devices and intelligent control from advanced countries to the West. Over the past few years, the China government's IoT growth plans have been significant and its ambitious objectives and IoT technologies have largely been implemented by power plants, transportation safety, financial services, defence and other industries. The IoT (IoT) mostly uses different means of transmission to gather, transmit and process object information and really realizes the relation of objects and the sharing of information between people and things as an extension of the internet [25]. 
A reflective flaw, which consists of malicious data inserted in a page, accompanies the request automatically and is returned to the client from the application. When you visit the vulnerability tab, the storage sort also returns malicious information. The greatest difference in security issues is that the server does not have to return the malicious code that has been sent. The browser collects and locally scans malicious data input. The object and dynamic upgrade page attributes are responsible for attacking the protection flaw. The malicious code does not return to the return page source; it is running straight away, though, is also a vital aspect of the vulnerability.

The initial web script is shown while you open the source code of the page. In the Wet HTML source code tab, this attack script will not exist. Protection weaknesses thus cannot be identified by the function approach that matches these two vulnerabilities in XSS, which impairs the automatic identification of vulnerabilities. As an IoT-convergence and mobile cars, the Internet of Vehicles (IoV) has arisen. Quan et al. have developed a vehicular network software-defined adaptive transmission control protocol. Their scheme offers a selective scheduling of different transmission monitoring policies by a Software Defined Networking controller and arranges with control units at the base stages in order to meet different vehicle needs.

Quan et al. have discovered a new prototype for the networking of the smart identification identifier and developed a tailored SINET approach that would allow crowds to cooperate for vehicle software. They showed that SINET-V has decent talents for supporting strong vehicle networks by experiments. In order to ensure effective and reliable delivery of big data, Cheng et al. reviewed numerous Vehicle Adhoc network technologies. They also addressed different methods for studying VANET features and results using big data. The development of IoT would result in a large amount of data, requiring massive information resources, storage space and bandwidth for communication[7]. According to Cisco's projection, up until 2020, the number of IoT devices could reach 50 billion[8] and by 2025 this figure will reach 500 billion[9].

It is also forecast that "data from man, computers and objects will be 500 zettabytes by 2019, but only 10.4 zettabytes will be available for the IP traffic of global data centres"[10]. Thus $45 \%$ of the information produced by IoT is collected, collected and analyzed near and at the edge of the network based on an observation made in [11]. The majority of IoT computers, by their very existence, have minimal resource capacity to calculate and store. In addition, most equipment is still powered by batteries. This poses a difficult challenge in developing safety protocols, which are easy to handle in computing and communicating costs, while retaining high security and the features required to develop procedures in IoT environments.

Das et al. [12] recently addressed a security protocol taxonomy which is highly necessary to protect an IoT setting. These units, for example in the year 2018 (6.2 Exabyte) generate large quantities of data which are expected to grow to 478 percent by the end of 2020 (30.6 Exabyte),[15]. This startling estimated 478 percent increase in data generation calls for a smart system planning and monitoring strategy. Many methods were proposed to address problems in the IoT model, but the standard network cannot handle such an immense number of linked devices and enormous data exploitation. Software Defined Network (SDN) is an innovative network technique that provides the diverse and rapidly changing networking 
with programmable planes like the control and the data plane. In different contexts, SDN and IoT implementation will satisfy planning and monitoring requirements.

\section{Existing Work}

Smart early-warning monitoring system enables network protection managers with detailed knowledge about device vulnerabilities to devise security measures that can efficiently deter disruptive hackers from stealing weaknesses and creating application harm. Vulnerability identification technologies have since highlighted numerous weaknesses in the advancement of network infrastructure and the development of new forms of vulnerabilities. Most bugs in lots are found in the current fault ability mitigation tools and there is no connection between faults. The simplistic risk overlay of vulnerability does not reflect the network's security status. In comparison, only known weakness was found and the suspected vulnerability was not addressed [26].

The information governance alert depends primarily on the technologies for imaging susceptibility. In general, vulnerability scan technologies can be classified in Host-based protection vulnerability, network-based vulnerability scan, target-based malware detection and application-based security auditing. And the most popular vulnerability scanning technology is network-based vulnerability scan. Host-based technologies of scanning protection vulnerabilities refers to the use for security assessments of an agent operating on a host device. The technology includes a search server for vulnerability and a weakness detector. The benefits of Host-based scanning vulnerability technologies include communication authentication, high accuracy of scanning, and simple management[22].

The network-based search technology for security vulnerabilities is primarily applicable to the business world. It uses various security vulnerability styles and characteristics. It generates network data packets with network servers and transmits them in many modes of propagation to different network destinations. If the deficiency is listed. The network-based intrusion check identification mechanism is identical to the downgrade process before the attack on the attack site by the hacker. In order to identify and interpret thrills of protection and security present in the operating system, security administrators or network managers routinely perform security vulnerability scans [21].

IoT is faced with many security problems, but we can sum up them in three broad categories: IoT, communications and security-related end apps. IoT requests collect a large volume of sensitive or private data from end nodes. For attackers and business rivals, such data provide a beneficial benefit. In addition, the reliability of IoT services such as personal, company and corporate depends heavily on the authenticity of information which has a deeper impact on their performance. The data provided by IoT end nodes shall be authentic and confidence for promising IoT service and application results Generic encryption algorithms cannot be used because of IoT node resource limitations. Thus, the need for complex encryption ciphers is negligible, such that resources-constrained nodes have optimum secrecy. A lot of cryptographic lightweight ciphers have recently been suggested. Any pioneers focused on modular block cipher hardware [20].

In various applications of IoT a compromise exists among expense, efficiency and security. For example, for RFID tags on electronic tickets security levels can be low, but there is a strong demand for low power and latency. The specification of 52 cryptographic 
algorithms was evaluated on the basis of a given parameter. These ciphers have been classified for specialized hardware end nodes. The authors established that they were vulnerable to Surface Chain Research attack due to the uncomplicated existence of most lightweight cryptographic ciphers. IoT-based Hash function, Elgamal and RSA faulty and time basis channel attacks have been explored and security measures proposed [19].

A broad structure for the investigation and assessment of arithmetic flaw threats on homomorphic encryption ciphers was another endeavour by $F$. Zhang et al. Many experiments have made use of physical features for data encryption and have proposeda specific way of creating keys for authentication purposes, Physical Unclonable Functions (PUFs). In reality it is extracted from PUFs using physical IC characteristics, the secrecy is not stored in memory. It is produced without using expensive hardware. Physical damage including such data theft, substitution and copying of nodes, etc. will undermine the validity of the end knots. It is essential to verify the authenticity of the data performance and credibility of the end nodes [17].

Therefore, certain compact authorisation mechanisms for IoT end nodes must be built. Three key approaches outlined in the literature are software, hardware and hybrid based static attestation. Side channel information is used to support the validity of end nodes without the use of advanced hardware in software-based certification techniques. Furthermore, it is divided into two major groups, namely recollection and timing. SWATT, Pioneer and SCUBA are part of the time foundation certification strategies. Although the strategies of memory-based certification includea multi-hop system between the provider and verifier, software and hardware architectures are used to protect future opponents, minimizing hardware amendments [15].

Hybrid certification methods with stable hardware cannot protect against the physical interference. Some of the methods of hybrid certification. As hybrid and software-based approaches are unprotected from physical attacks due to stolen passwords, because the prover can be simulated. Only hardware certificates can protect physical attacks. Hardware based certification procedures depend on functions such as TPM or SGX that cannot be found on end machines which are resource-based. IoT uses special lightweight hardware features for this purpose. The initial swarm certification methodology proposed was SEDA. SANA submitted another swarm certificate proposal [14].

In IoT the end nodes often link and dynamically exit their swarm much as in ad hoc vehicle networks, which makes proof of a swarm device more difficult. Many of the above static attest methods verify rather than perform the validity of binaries. By controlling the execution flow of the Program in IoT-embedded end nodes, C-FLAT and LOFAT provided valid attestations. Devices and users in the IoT ecosystem need safeguards like authentication and access control and their connectivity interchanges. However, device permission must be authenticated in advance. The diverse IoT ecosystems require the development of different terminal nodes, diverse network infrastructure and especially limited IoT resources, easy authentication and authorization processes. Since a trustworthy third party does not operate in a distributed IoT setting, authentication mechanism is mandatory for an IoT system [11].

In a heterogeneous IoT environment, data collectors as well as data holders need to be mutually inspected before gathering and transmitting data to each other. C. Su et al. emphasized the RFID encryption protection and protection problems among tag and the 
reader. Some researchers stressed that IoT major applications such as smart health, grids, and Intelligent transportation System require deliberate unlikability and anonymity (IoV). The novel technique cross-domain authorization protocols are required by dynamic IoT devices that need regular location changes. The IoT Access points from edge devices which are transported across multiple networks and run by diverse IoT systems combine a massive amount of data [12].

Forensic evidence, political or moral problems and concerns concerning privacy are few issues related to the development, transmission and use of data. Since data collection and processing are large quantities, it creates an additional issue of data ownership, with little concerns, such as How to standardize data management as a product? To decide who owns/holds the data? Is it possible to trade data? Due to these issues, legal obligations are invoked. The rights of data owners must be to authorize and revoke the permission for data processing. Data owners can use the context-based granular authorisation to share the amount of data that can be exchanged with the IoT ecosystem [18].

A general framework for digital forensic research forforms a homogeneous digital research procedure, DFIF-IoT was submitted. FAIoT has been suggested to simplify the protocol for compiling, analyzing and preserving data. It adopted a method of 1-2-3 zones and the next best possible thing model. Another important thing to be considered during the forensic examination of IoT is the protection of privacy when examining and correlating composed documentation that could include individual personal data. IoT-conscious privacy Forensic is an attempt to propose an IoT forensic model that allows privacy. Evidences can be gathered from the ProFIT model using neighbouring devices which contributes to the very precise level of reconstruction of the crime scene context. From the literature we have investigated, we can conclude that IoT forensics are still developing and that most of the investigators are extending conventional IoT forensics approaches.Even if conventional forensic methods are to some degree available in IoT, there is still a lack of a full system for IoT forensics [13].

\section{Proposed System}

The attacker network has the basic features of the addressed graph by the study of the insecurity threat weighted graph in the IoT environment. The design of the attack graph could correspond to the directed graph's cross-section process. The reverse part of the guided diagram primarily consists of two algorithms: the first-degree search cross and the secondbroad search cross-section, and the second-degree cross-sectional part of the attack diagram is the acquisition of all current state relationships. Consequently, the node cross-section is more appropriate for the first width-scale random search.

Again, from initial state node, with a forward search algorithm begins and scans the next state node which can be attacked. If no new node is open, the search ceases and the current path of search ends. From the viewpoint of the attacker, the forward search algorithm does not indicate a certain target status, because the search spectrum is relative wide. The backward search algorithm identifies first of all the goals which could be attacked in the network structure in view of safeguarding the particular property, that is, looking back from the target state to find the previous condition which leads to the target state, looking up and reaching, finally, the original status node or suspends well beyond highest limit of attacker hops. 
The reverse search algorithm has a simple objective and remove state nodes, which are not linked to the target state. The attack graph developed is therefore small and shows only the graph-based sequence that attacks the identified target and can help protection managers tend to focus on those attack paths. The important IoT services are clustered and decentralized in such dynamic network settings. Particularly in recent years, cloud infrastructure has developed, distributed network resource storage is highly frequent. This uncertainly and diversifies the attack goal. From the point of view of the assailant, although the crossover is large and the attack map created is more complex, both attack triggers and targets within the network are feasible.

The recursive proposed algorithm cannot specify all sequences of attack within the system, but it can reduce the graph for attacks for targets specified and provide the most concerned safety status for the network resources. The node of attack entered the attack queue and started it. Using a host to look for neighbouring attacks and locate the attack route using the front search algorithm. The network is applied to the existing sequence of attack each moment a node will successfully penetrate. The attack sequence is scanned backwards to delete redundant nodes and foreign nodes and the current sequence of attack is decreased if the target nodes enter the attack node or the number of attacks surpass the maximum value of this sequence range.

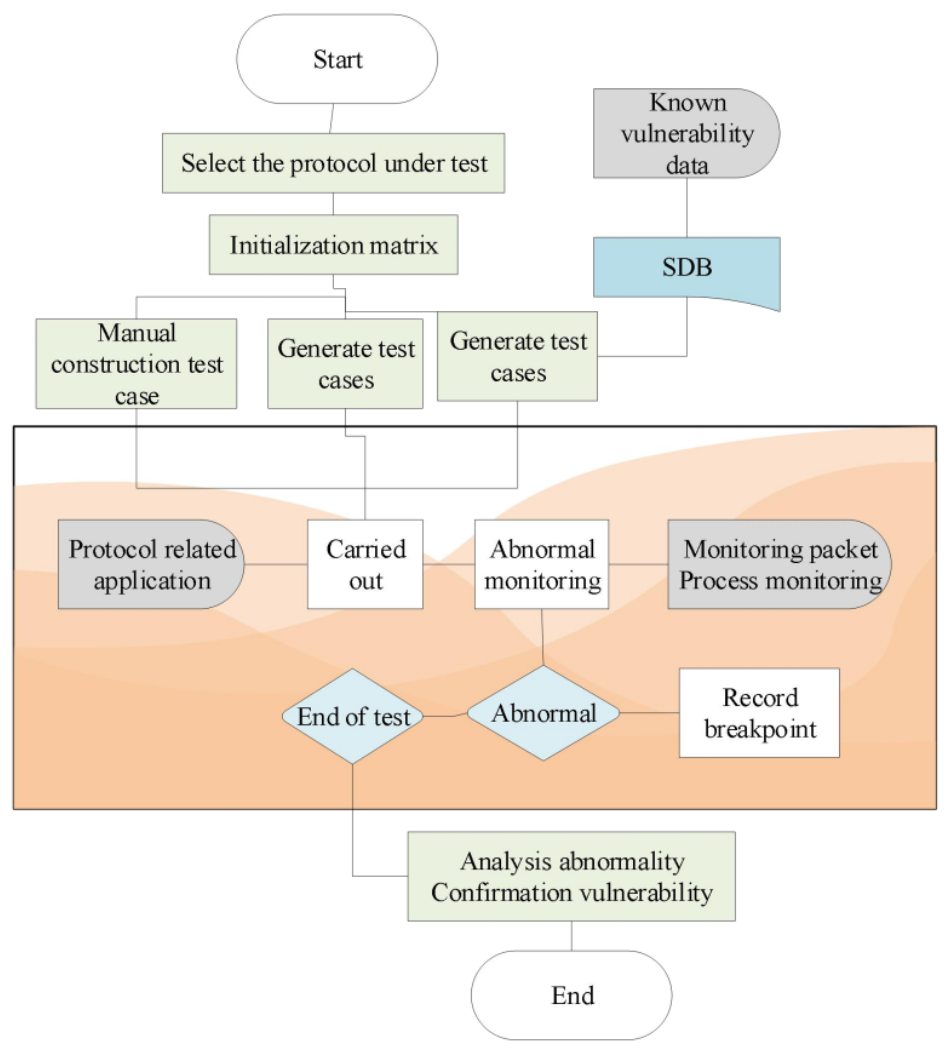

Figure 2. Flow model of proposed approach

If the target node is not hit, and the quantity of attacker hops is less than the value set, loop 2 will be carried out before all the attack nodes in the queue finish the scan. The PR value of the current page is calculated, each page's PR value is calculated, the weight of the current page is calculated by the high reserve weight and the Access Link. The stronger the 
PR rating, the greater the connection or web page entry, the more significant the web page and the greater the PR rating.

$$
P R(X)=(1-e) / M+e *\left(\frac{P R\left(X_{1}\right)}{D\left(X_{1}\right)}+\cdots \frac{P R\left(X_{m}\right)}{D\left(X_{m}\right)}\right)
$$

The estimation model shall be extended to the calculation of the attack graph status node weight.

$$
S(T)=(1-e) / M+e *\left(\frac{R\left(T_{1}\right)}{D\left(X_{1}\right)}+\cdots \frac{R\left(X_{m}\right)}{D\left(X_{m}\right)}\right)
$$

where, $M$ represents the total of all configuration nodes in the graph $S(T)$ of the attack representing the strength of the graph node $P R\left(X_{i}\right)$ means that $R$ of the state node $N$ is marked as the grade arc, with $\mathrm{C}(\mathrm{Si})$ as the weight of the state node $S$ representing the number of arcs of departure as $0<e<1$. $e$ is typically 0.85 . In a risk estimate, the cumulative failure in critical attack paths is calculated. Let the likelihood that perhaps the state node Si penetrates the next state node quo1 be $T_{k}, 1$ as well as the possibility that the state node $\mathrm{Si}$ is the first state node:

$$
P_{0, j}=\prod_{l=0}^{j-1} P_{l, l+1}
$$

If the State node loss is then the average attack loss on the attack graph is: If the State node loss is $M i$

$$
M=\sum_{j=0}^{m}\left(M_{j}, P_{0, k}\right)
$$

$M$ is the threat value of the attack graph's critical path. It also acts as a safety metric for the whole network. It offers detailed benchmarks for the security state of the targeted network, guiding security management staff to implement effective and reliable security policies.

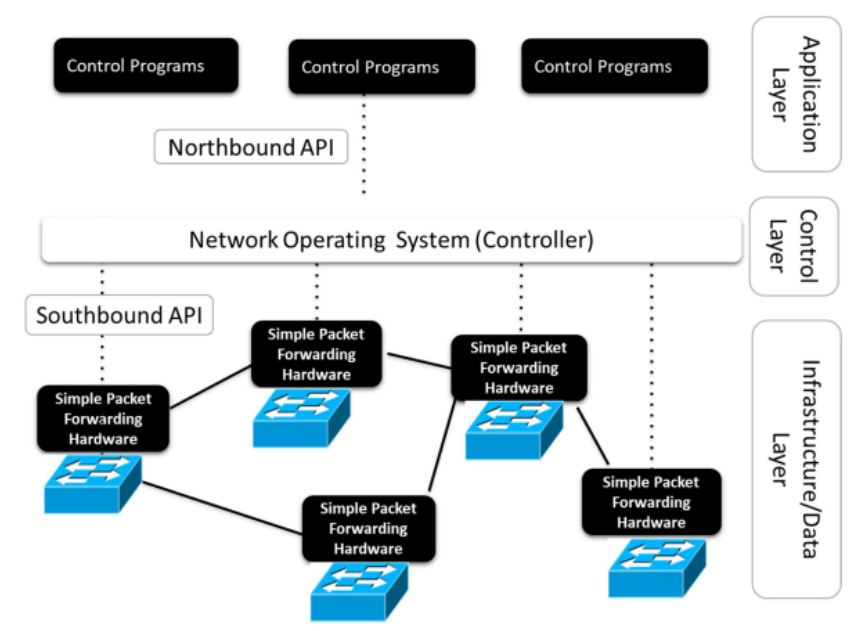

Figure 3. Layered architectural overview 
Throughout the IoT environment, advance warning security flaws are strongly connected to the receipt of external data. The use of unverified web-based external input data is the key to vulnerability generation. Also recognized as tainted data is external input data. The method of monitoring the transmission and allocation of exploit information is considered stain transmission assessment.The analysis of the spread of stubble can usually be divided into the analysis of stain spread and dynamic stain propagation analysis. Statistical analysis uses source code and needs syntax and semantized characteristics to be extracted. Source code in many cases is difficult to get and the high false positive rate is problematic. Flexible stain transmission assessment involves technological innovations such as fully automated labelling of smudges, dynamic propagation and able to monitor of stains, and main data recording.

When the program is executed, it immediately detects the contamination and execution of the data. The identification speed is faster, and the suggested technique does not necessitate the application source. Consequently, this document applies the concept of flexible blemish study constructed in the procedure of smart emergency alert security vulnerabilities mining identification, which usually entails the dynamic transmission andmonitoring advanced technologies of stains. According to the review above there has to be at least one way from the untrusted external input to hazardous operations if there is an intelligent early warning risk in the IoT environment.

The customer input taint data is the intelligent early warning vulnerability object system and attribute of the dynamic update page through data transfer, encoding, filtering and purification operations, and the execution succeeds or causes an abnormality in the intelligent emergency alert finding vulnerabilities in the IoT environment. Vulnerability indicates that there is.

Smart early warning vulnerabilities identification is then closely linked to stain data in the device propagation trajectory in the IoT environment. Models for dynamic blot spreading include the application of ink, stain diffusion and examination of stain. The customer test script incorporates smears, variable assignment, sorting and filtering methods. The implementation and variations to the design document shall be regulated by Stain Verification. The blot data is split, moved, encoded and filtered within the software in the IoT environment and the function will be entered as a parameter.

The distributions of the upper and lower surfaces reflect blot data and an intelligent vulnerability sensing warning. The detection shows that during the inner spread of the program the plot data is labelled. The tracking curve and the logging of bullet data has a curve number to detect the exposure of intelligent early warning such that the sensitivity of the early warning sensation is of little importance. The input- and output point is obtained by hybrid drive detection in protocol-driven mode to enhance the algorithm's detection performance. Since the smart early alert is a connected and stateless object-focused protocol, only the website's static content is easy and scalable.

Therefore, features are more efficient. When the stain data is inserted into your tab, the screen output point will cause the machine to call the parser to respond to the special feature in the test document, and it will inevitably decrease performance by the dynamical execution of your page. This hybrid drive detection process is seen to be more effective than with a single event-driven detection. The input- and output point is obtained by hybrid drive 
identification in protocol-driven mode to enhance the algorithm's detection accuracy. Since the smart early alert is a connected and lawless object-focused method, only the website's static content is easy and scalable. Therefore, features are more effective.

If the spoils have been inserted in the page, the display point inevitably needs to hear the machine calling the parsing motor, and dynamic page performance would undoubtedly lead to a reduction in quality. Analysis reveals that this approach is more effective than for one event-driven identification. This method is more efficient. Get high precision identification and reliability of detection. The vulnerability detection algorithm, based on the counterexample, integrates data stream analysis with the effects of restriction analyse to jointly conclude the emergency alert vulnerability detection in order to solve the shortcomings of the static detection process for advance warning weaknesses by means of data stream analysis. If the current process' entry parameters are indexed in the limit state, the search result cannot be specified.

\section{Results and Discussion}

The test results illustrate the basic facts on the host vulnerability: Throughout the local network the attacker will normally enter the web site and the FTP server. The firewall cannot detect the attacker's actions. The original assertion of the intruder is his own server $\mathrm{H}$. The intruder uses the $\mathrm{H}$ platform to bypass remote proxy server and FTP server weaknesses to infiltrate and increase privilege but instead uses the server as a stepping stone to keep penetrating the user host or intranet network server. The mixture of forward-looking and backward-looking search in the IoT context is used in the graph-based generation process. As is seen. With the threat chart development, the weakness attacker will plateau.

The residual entities in the diagram have a reproductive and evolution mechanism, as the deficiency shifts, using the smart emergency alert method. Suppose the host H2 is the MySql database server and stores key intranet data. The intruder also has the prime goal of just such a computer, and the most important and 10 is important. The security assessment of each State node shows, that an attacker uses the HO network to manipulate the web server and FTP server vulnerabilities to infiltrate and enhance privilege; and that the servers are used as a springboard such that the user host or intranet client server continues to penetrate.

The attack on the network Take the weight of the node as the chance of success of the node attack and use the risk of attack formulation of the smart early warning vulnerability. The solution is S0-Si, Pi is the likelihood of attack and Pi is the expected loss of sequence. The cumulative failure expectations for the Crisis Course were estimated based on the results from the experiments. The risk value is the metrical value of the operational system which is used to represent the total security state of the present network, and a measured security factor allows different information security. 

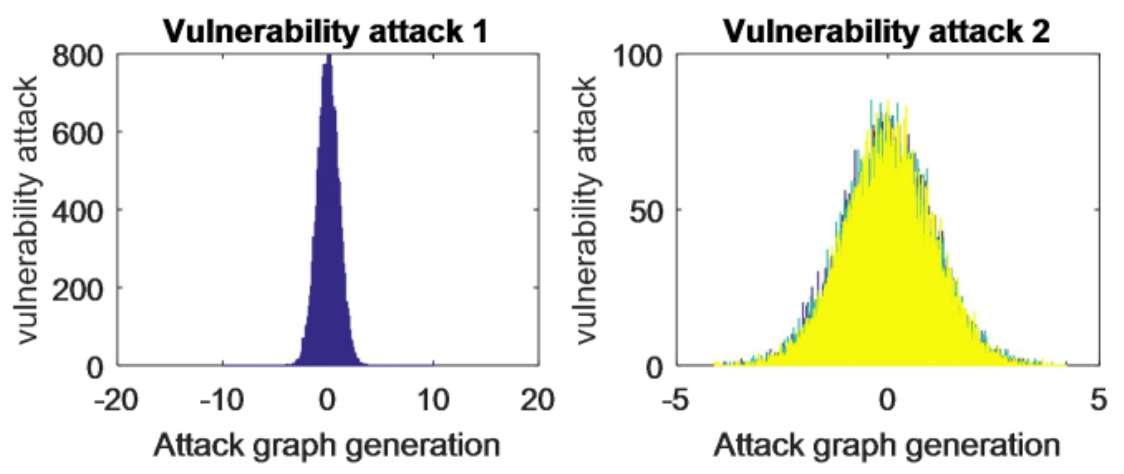

Figure 4. Performance Analysis

The failure expectation of the threat path is 12.8. The critical path is the value of the attack risk value. The risk value is 12.92 . The condition is benchmark and gives Network Safety Administrators guidance. The Internet of things uses C\# to enforce protocol-driven crawling and uses a standard attack series in the IoT environment for the effective attack probability for each node in the crucial attack line. The result is seen in terms of input and output points in order to achieve the IoT evaluation. When using C\#-enabled open-source software, it replicates user operations on webpages, causes events, and analysis of the layout changes of the DOM board by modifying the window and the DOM objects on this page.

The hijacking feature of the authentication module must provide in the answer data package the code for the hijacking function. The reporting device is used in the experiment to filter and substitute the data packet and written the centre of the filter file. This paper builds an advance warning mitigation algorithm in the IoT ecosystem for a local test website, extracts the vulnerability published by the vulnerability publishing forum, and creates a comparable environment for vulnerability recurrence in order to check the efficacy of an algorithm. The smart contact Early Detection Risk Identification in the IoT setting is primarily split into 2 groups to maintain the authenticity of the software verification: 1 . no UI processing and cleansing mostly in case of failure of code writer, ignoring limitations induced by user input filters; 2. Sorting and encoded limitations, particularly for code writers with inadequate knowledge of XSS, and logical problems in the encoding process.

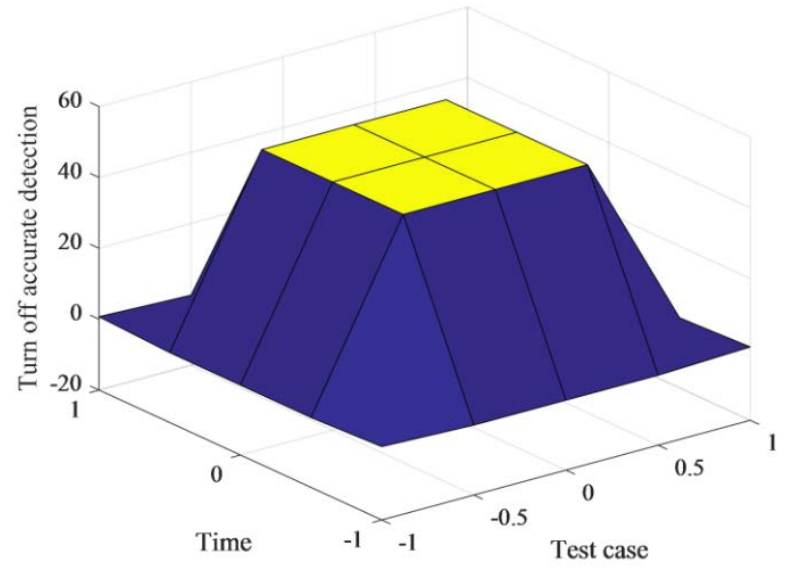

Figure 5. Attack detection modelling

The experimental findings show that un-filtered exploits are marginally better performed because the hybrid operator goblin can scope the page dynamically, which is 
useful in gaining more input which output points, and has clear benefits in identifying input points which are overlooked by programmers. The identification effect is easier when identifying the filtered vulnerability. The initial research is more efficient since the test cases chosen are better. Due to the diverse screening and purification techniques used to identify vulnerability in early warning of intelligent communication, this variety contributes to identification.

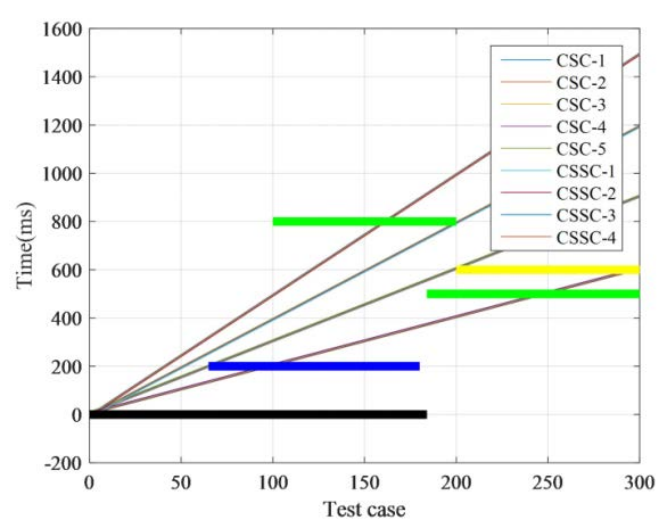

Figure6. Performance comparison with other approaches

The difficulty is enhanced and the recovery rate of the two instruments is not especially high. The average time the tests have been performed requires time to detect the vulnerability, manual checking, better performance of the identification, and automation is to some degree resolved. From the experimental data, it can be shown that when detecting nonfiltered vulnerabilities, the efficiency is marginally lower, since the hybrid driver crawler can automatically crawl a page, which is useful to get more input and output points. The identification effect is easier when identifying the filtered vulnerability.

The preliminary research is more efficient since the test cases chosen are better. Due to the difference in complex model framework filtering and purifying approaches, which are intelligent early-warning vulnerability identification, this diversity contributes to the detection. The complexity is raised and thus the retrieval rate for both instruments is not especially high; the time used to validate them is the amount of time used to find the flaw and manually to verify the accuracy, the detection effectiveness is enhanced and the automation is to some degree solved. Finally, an advance warning vulnerability algorithm for the identification of mines is used for intelligent communication.

The principal detection material is the number of vulnerabilities identified, the detection precision and the retrieval rate and the overall time spent on detection reflecting the detection method's accuracy and coverage. The findings of the evaluation are demonstrated. It is a dispensing of the time that the BVC detects quickly and accurately. It is indeed a transmission scheme of the time that the BVC only detects quickly. We have performed two loop analysers, one on a loop analysis tool based on reverse path and the other on a conventional loop analyzer using an extension / narrowing procedure in order to measure the effect of various cyclical analysis techniques on the BVC detection effect. The LAR and LAT are respectively named for the two loop analysers. 


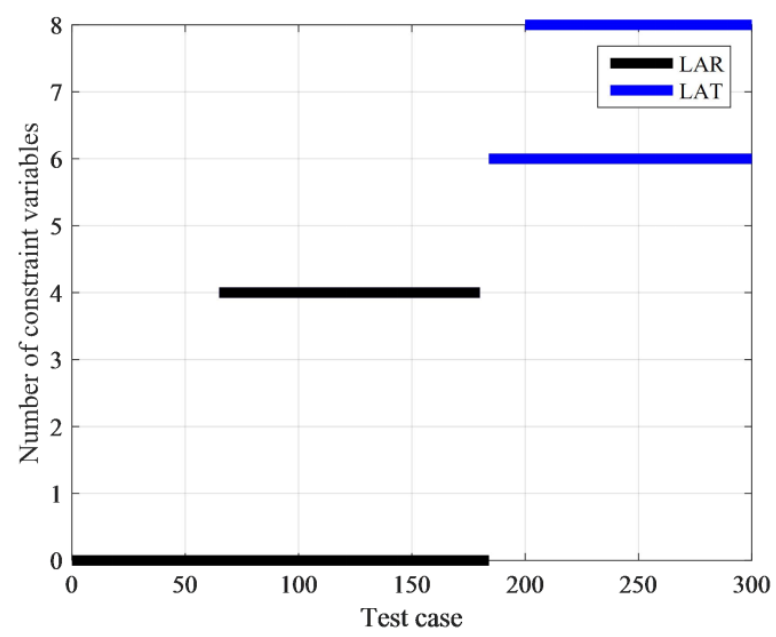

Figure 7. Constraint variable comparison

As an intermediary code translated from the front end is in the context of a compiler LLUM, the assignment argument does not reverse, so the analytical accuracy of a standard approach is not enhanced by the reverse assignment method. This really is the examination result. BVC has no false positive for CSC and BVC has 14,5\% for CSSC which means that the limitation of state security identification with an enhanced scope restriction and the control limit will efficiently use the control restrictions to measure the constraint condition of this paper correctly. First, LAR and LAT were tested independently for their analytical accuracy. Figure indicates the number and limit variables of the x-axis of both analysers and the accuracy of $y$.

Data shows that perhaps the $\mathrm{x}$ axis means that 233 restriction parameters in all 271 test cases as compared with the LAT test are described by the LAR test results. In the LAR data, the range of 184 limit variables is higher than in the LAT results. Precisely, LAR increases by more than 50 percent the analytical precision of the constraint vector. To measure the identification effect of various methods of restriction generation, we use 2 restrict state security controls, one is a restricted state security control system, the other is a basic state security checker, two restrictions. The CSC and CSSC are referred to as State Protection Checks.

The majority of the BVC was unchanged in the next trials, except that separate restriction state controls were checked. The static detection approach will, as shown, increase the accuracy and effectiveness of buffer alert vulnerability detection by the Smart Alert Vulnerabilities Validation with five distinct CSC and four separate CSSC. In specific, detection rate performance can be increased through user intervention using an example buffer alert weakness.

\section{Conclusion}

The whole document analyses the current techniques of IoT safety evaluation. The standard security network evaluations mostly include overlapping vulnerability quantification and failing to carry out multiple regression of deficiencies in the system. The article examined a graph-based interaction study of the Distributed device and an attack graph creation methodology for the information security evaluation process. The weight estimation model is implemented and the way to identify the main attack path using the node weight is 
suggested. The procedure is finally suggested. In order to evaluate IoT safety status, the essential attack route is used and a comprehensive assessment plan for the protection status of IoT is given. Research study evaluates the formation of the Intelligent alerttheory under IoT and suggests an Emergency Alert Susceptibility Dynamic Propagative Model for Smart Communications under IoT. Static early warning vulnerability identification system based on the IoT counterexample. As a hierarchical analysis tool, this system detects potential premature buffer alert vulnerabilities using stream detecting and context-sensitive quick detection and then performs effective journey and context-sensitive detection, leading to the removal of quick detection effects. The latest false positives have a particular route to buffer alerts. The intelligent early warning vulnerability algorithm seeks the input and output point page level to push the crawler with the protocol. The injection stage is compared with the current tools of detection in the experimental setting, using the event-controlled crawler and proving the proposed algorithm experimentally. The intelligent contact can efficiently predict an early identification of weakness in the IoT system. The next step would be to apply the new system to the current network area. The prototype structure is proposed for topology and inspection and the security parameter measurement is carried out on the experiment system.

\section{References}

1.Anwar A, Mahmood A N, Tari Z. Ensuring Data Integrity of OPF Module and Energy Database by Detecting Changes in Power Flow Patterns in Smart Grids[J]. IEEE Transactions on Industrial Informatics, vol.13, no.6, pp.3299-3311, 2017.

2. Bhilare S, Kanhangad V, Chaudhari N. A study on vulnerability andpresentation attack detection in palmprint verification system[J].Pattern Analysis \& Applications, vol.21, no.3, pp.769-782, 2017.

3. Braeken, P. Porambage, M. Stojmenovic, and L. Lambrinos, “eDAAAS: Efficient distributed anonymous authentication and accessin smart homes," International Journal of Distributed Sensor Networks,vol. 12, no. 12, pp. 1-11, 2016.

4. Chen X, Lin W, Yu W. A General Framework for Hardware TrojanDetection in Digital Circuits by Statistical Learning Algorithms[J].IEEE Transactions on Computer-Aided Design of IntegratedCircuits and Systems, vol.36, no.10, pp.1633-1646, 2017.

5. Challa, M. Wazid, A. K. Das, N. Kumar, A. G. Reddy, E. J. Yoon, andK. Y. Yoo, "Secure Signature-Based Authenticated Key EstablishmentScheme for Future IoT Applications," IEEE Access, vol. 5, pp. 3028-3043, 2017.

6. Das, "A random key establishment scheme for multi-phasedeployment in large-scale distributed sensor networks," InternationalJournal of Information Security, vol. 11, no. 3, pp. 189-211, 2012.

7. Fan, S.-Y. Huang, and Y.-L. Lai, "Privacy-enhanced data aggregation scheme against internal attackers in smart grid,” IEEE Transactionson Industrial informatics, vol. 10, no. 1, pp. 666-675, 2013.

8. Fernandes, A. Rahmati, J. Jung, and A. Prakash, "Security implications of permission models in smart-home application frameworks,”IEEE Security \& Privacy, vol. 15, no. 2, pp. 24-30, 2017. 
9. Ge, P. Zeng, R. Lu, and K.-K. R. Choo, "Fgda: Fine-grained dataanalysis in privacypreserving smart grid communications,” Peer-toPeer Networking and Applications, vol. 11, no. 5, pp. 966-978, 2018.

10. He, J. Bu, S. Zhu, S. Chan, and C. Chen, "Distributed Access Controlwith Privacy Support in Wireless Sensor Networks,” IEEE Transactionson Wirless Communications, vol. 10, no. 10, pp. 3473-3481, 2011.

11. Koya and P. Deepthi, "Anonymous hybrid mutual authenticationand key agreement scheme for wireless body area network,” ComputerNetworks, vol. 140, pp. 138-151, 2018.

12. Liu, Y. Li, J. Qu, and Y. Ding, “A lightweight pseudonym authentication and key agreement protocol for multi-medical server architecturein tmis.” TIIS, vol. 11, no. 2, pp. 924-944, 2017.

13. Li, M. H. Ibrahim, S. Kumari, A. K. Sangaiah, V. Gupta, and K.K. R. Choo, “Anonymous mutual authentication and key agreementscheme for wearable sensors in wireless body area networks," Computer Networks, vol. 129, pp. 429-443, 2017.

14. Luo, Y. Luo, Y. Wan, and Z. Wang, "Secure and efficient accesscontrol scheme for wireless sensor networks in the cross-domain contextof the IoT," Security and Communication Networks, vol. 2018, pp.1-10, 2018.

15. Le, S. Lee, I. Butun, M. Khalid, R. Sankar, M. Kim, M. Han,Y.-K. Lee, and H. Lee, “An Energy-Efficient Access Control Schemefor Wireless Sensor Networks based on Elliptic Curve Cryptography,”Journal of Communications and Networks, vol. 11, no. 6, pp. 599606,2009.

16. Li, Y. Han, and C. Jin, "Practical access control for sensor networksin the context of the Internet of Things,” Computer Communications,vol. 89-90, pp. 154-164, 2016.

17. Patel T B, Patil H A. Cochlear Filter and Instantaneous FrequencyBased Features for Spoofed Speech Detection[J]. IEEE Journal ofSelected Topics in Signal Processing, vol.11, no.4, pp.618-631,2017.

18. Peng S, Liu P, Han J. A Python Security Analysis Framework inIntegrity Verification and Vulnerability Detection[J]. IEEETransactions on Industrial Informatics, vol.24, no.2, pp.141$148,2019$.

19. Porambage, A. Braeken, C. Schmitt, A. Gurtov, M. Ylianttila, andB. Stiller, “Group Key Establishment for Enabling Secure MulticastCommunication in Wireless Sensor Networks Deployed for IoT Applications,” IEEE Access, vol. 3, pp. 1503-1511, 2015.

20. Riedl, V. Grascher, and T. Neubauer, "A secure e-health architecturebased on the appliance of pseudonymization.” JSW, vol. 3, no. 2, pp.23-32, 2008.

21. Rottondi, G. Verticale, and A. Capone, "Privacy-preserving smartmetering with multiple data consumers,” Computer Networks, vol. 57,no. 7, pp. 1699-1713, 2013.

22. Satpathi K, Yeap Y M, Ukil A. Short-Time Fourier Transform BasedTransient Analysis of VSC Interfaced Point-to-Point DC System[J].IEEE Transactions on Industrial Electronics, vol.65, no.5, pp.4080-4091, 2018. 
23. Sahi, H. Abbas, K. Saleem, X. Yang, A. Derhab, M. A.Orgun, W. Iqbal, I. Rashid, and A. Yaseen, "Privacy preservation ine-healthcare environments: State of the art and future directions,” IEEE Access, vol. 6, pp. 464-478, 2017.

24. Seol, Y.-G. Kim, E. Lee, Y.-D. Seo, and D.-K. Baik, "Privacypreserving attribute-based access control model for xml-based electronic health record system,” IEEE Access, vol. 6, pp. 9114-9128, 2018.

25. Tsai, C. M. Yu, H. Yokota, and S. Y. Kuo, "Key Management inInternet of Things via Kronecker Product," in 22nd Pacific Rim International Symposium on Dependable Computing (PRDC’17), Christchurch,New Zealand, 2017, pp. 118-124.

26. Tian, N. Zhang, Y.-H. Lin, X. Wang, B. Ur, X. Guo, and P. Tague,“Smartauth: Usercentered authorization for the internet of things," in26th fUSENIXg Security Symposium (fUSENIXg Security 17), 2017,pp. 361-378.

27. Wei X U, Leng J. Simulation of Potential Vulnerability SpilloverDetection Under Network Active Protection[J]. ComputerSimulation, vol.183, no.993, pp.190-202, 2018.

28. Yang L, Hu S, Zomaya A Y. The Hierarchical Smart HomeCyberattack Detection Considering Power Overloading andFrequency Disturbance[J]. IEEE Transactions on IndustrialInformatics, vol.12, no.5, pp.1973-1983, 2017.

29. Yasinzadeh M, Akhbari M. Detection of PMU spoofing in powergrid based on phasor measurement analysis[J]. Iet GenerationTransmission \& Distribution, vol.12, no.9, pp.19801987, 2018. 